\title{
Evaluation of creatine kinase and liver enzymes in identification of severe dengue
}

\author{
Saiful Safuan Md Sani ${ }^{*}$, Winn Hui Han ${ }^{1}$, Mohamad Adam Bujang ${ }^{2 *}$, Hui Jen Ding ${ }^{1}$, Kiah Loon Ng \\ and Mohd Azizuddin Amir Shariffuddin ${ }^{3}$
}

\begin{abstract}
Background: Existing biomarkers such as AST, ALT and hematocrit have been associated with severe dengue but evidence are mixed. Recently, interests in creatine kinase as a dengue biomarker have risen. These biomarkers represent several underlying pathophysiological processes in dengue. Hence, we aimed to assess AST, ALT, CK and hematocrit in identification of severe dengue and to assess the correlational relationship amongst common biomarkers of dengue.

Methods: This was a retrospective cohort study of confirmed dengue patients who were warded in Kuala Lumpur Hospital between December 2014 and January 2015. CK, AST, ALT, hematocrit, platelet count, WBC and serum albumin were taken upon ward admission and repeated at timed intervals. Composite indices based on admission AST and ALT were analyzed. Correlation coefficients and coefficients of determination were computed.
\end{abstract}

Results: Among the 365 cases reviewed, twenty-two (6\%) patients had severe dengue. AST and ALT were found to be good at identification of severe dengue. The AST ${ }^{2} / A L T$ composite index was the most accurate (AUC 0.83; $95 \% \mathrm{Cl} 0.73$ - 0.93). Optimal cutoff was 402 with a sensitivity of $59.1 \%$ (95\% Cl: $36.4-79.3 \%$ ) and specificity of $92.4 \%$ (95\% Cl: 89.1 - 95.0\%). Modified cutoff of 653 had a sensitivity of $40.9 \%$ (95\% Cl: 20.7 63.7\%) and specificity of $97.4 \%$ (95\% Cl: 95.1 - 98.8\%). Our analyses also suggested that several underlying biological processes represented by biomarkers tested were unrelated despite occurring in the same disease entity. Also, markers of plasma leakage were discordant and AST was likely hepatic in origin.

Conclusions: The composite index $\mathrm{AST}^{2} / \mathrm{ALT}$ may be used as a marker for identification of severe dengue based on admission AST and ALT, with two choices of cutoff values, 402 and 653. AST is most likely of liver origin and CK does not provide additional value.

Keywords: Severe dengue, Creatine kinase, AST, ALT, Identification, Sensitivity, Specificity

\section{What is already known about this topic?}

It is already known that elevations of both AST and ALT levels are associated with bleeding and dengue hemorrhagic fever. Literature on creatine kinase in dengue is inadequate. Hematocrit and serum albumin are considered as markers of plasma leakage.

\footnotetext{
* Correspondence: dcicantab5@gmail.com; adam@crc.gov.my

'Department of Medicine, Hospital Kuala Lumpur, Jalan Pahang, 50586 Kuala Lumpur, Malaysia

${ }^{2}$ Biostatistic Unit, National Clinical Research Centre, 1st Floor, MMA Building,

124 Jalan Pahang, 53000 Kuala Lumpur, Malaysia

Full list of author information is available at the end of the article
}

\section{What does this article add?}

This study provides evidence that AST and ALT taken upon admission, through composite index $\mathrm{AST}^{2} / \mathrm{ALT}$, are good parameters in identifying severe dengue regardless of types of severe dengue. AST is most likely of liver origin and CK does not provide additional value as a severity marker. Additionally, two markers of plasma leakage (hematocrit and serum albumin), do not correlate with each other.

\section{Background}

Dengue infection is endemic to the tropical and subtropical regions of the world and is predicted to 
worsen and spread to wider latitudes [1]. In 2014, for the first time in history, the number of confirmed dengue cases in Malaysia breached the 50,000-case mark [2].

Like any other disease, dengue has biomarkers which are used to identify dengue infection, act as surrogates for underlying pathological process or identifying severity of infection. Current established biomarkers in clinical practice are hematocrit (Hct), platelet count, white blood cell (WBC), serum albumin and alanine aminotransferase (ALT). Recent studies and the 'breakbone' feature of dengue have sparked an interest in creatine kinase (CK). It has been shown in a small cohort study involving adult dengue patients $(n=39)$ that CK can be elevated, median 837 (range 194-3832 U/L) [3]. It has been further documented that any level of elevated CK taken after 48-96 h from onset of illness, was indeed associated with a more severe disease (ie. dengue haemorrhagic fever, DHF) by Cox proportional hazard regression analysis, HR 6.98, 95\% CI 2.34 - 20.85, $p<0.001$ [4].

Hepatitis, represented by elevated levels of aspartate aminotransferase (AST) and ALT, has a specific pattern. It is well-documented that AST is elevated to a higher level [5-9] and in a larger proportion [10-13] of patients as compared to ALT. It has also been shown that AST rises and reduces before ALT [8]. Although AST has been found to be elevated greater than ALT, its origin, musculoskeletal or hepatic, has not been proven $[8,11]$. The association of transaminitis with disease severity has been found to be inconsistent. One group of investigators in Thailand [9] showed that AST but not ALT was associated with DHF, whilst another group showed both liver enzymes were significantly associated with severe cases (AST $p<0.001$, ALT $p=0.003$ ) [14]. Both AST and ALT have been claimed to be significantly correlated with clinical plasma leakage but the strengths of correlation were not reported [12]. A later study in Vietnam showed that both AST and ALT were associated with shock [8]. They found that AST and ALT levels during the critical phase were significantly higher in dengue patients who experienced shock compared to those without shock $(p<0.01)$. They also found that transaminitis correlated weakly with the nadir thrombocytopenia (Spearman $r-0.2$ to -0.3 ; both $p<0.05$ ). One study in Singapore showed no association between transaminases and fatality, (logistic regression analysis (fatal outcome), AST $p=0.14$, ALT $p=0.11$ ) [15], and another showed transaminases lacked the discriminatory function to classify dengue according to severity by the WHO 2009 classification (AST
AUROC 0.62 (95\% CI:0.57 - 0.67) and ALT AUROC 0.60 (95\% CI: $0.54-0.64)$ [11]).

Widely available and practical biomarkers (elevated CK [4], liver transaminases [10-12, 14-17], Hct [17] and low serum albumin $[4,14,17]$ and platelet count $[12,14,16,17])$ have been shown to be associated with more severe disease. These biomarkers each represent a specific underlying pathophysiological process. Hct and serum albumin have long been believed to be representative of plasma leakage [18]. Platelet count and WBC have been shown to be good at reflecting disease course [19]. CK reflects muscle involvement and ALT the liver. Pathophysiological processes occurring in a single disease ought to correlate with each other. Furthermore, as these biomarkers have been shown to be associated with severe disease, a certain degree of correlation is expected amongst them. However, in our clinical observation, we have found them to be discordant with each other. Quantifying their correlation coefficients which measure the strength of those relationships, if any, may clarify their associations with each other.

Hence we endeavoured to evaluate the function of elevated CK, AST and ALT in the early identification of severe dengue. Secondly, we looked to clarify and quantify correlations amongst biomarkers: CK, AST, ALT, Hct, serum albumin, platelet count and WBC. This in turn will clarify whether AST is predominantly liver or muscle in origin.

\section{Methods}

\section{Ethical considerations}

The study was approved by the Malaysian Research Ethics Committee, Ministry of Health of Malaysia (Research ID NMRR-14-1543-23635). Our report is based on the Standards for Reporting of Diagnostic Accuracy (STARD) 2015 guideline [20].

\section{Study design}

This was a retrospective cohort study of adult patients with confirmed dengue infection who were admitted to Kuala Lumpur Hospital. Sample recruitment was conducted from December 2014 until January 2015. Sampling was universal. Inclusion criteria were patients $\geq 18$ years old and presentation which satisfied the WHO 2009 criteria [21] for suspected dengue. In Malaysia, patients with dengue infection are admitted when they manifest severe dengue characteristics, warning signs, have underlying co-morbidities or have healthcare accessibility issues. Our centre adopts these based on recommendations of our own national clinical practice guidelines which in turn have absorbed many of WHO 2009 clinical guidelines recommendations and adopted fully WHO 
2009 criteria. In addition, cases selected were required to have the presence of acute dengue viral infection confirmed via presence of NS1 antigen and/or hightitre level of IgG from an admission serum sample. Cases were excluded if they: 1) had underlying liver diseases, or 2) had received recent (within 5 days) intramuscular injections. Cases were subsequently classified into severe and non-severe dengue.

Case notes were reviewed and data, including baseline characteristics, clinical parameters and laboratory findings, were collected using a pre-defined data collection form. All patients received standard management adhering to the 2010 Malaysian Clinical Practice Guideline on Management of Dengue Infection in Adults [22] and the 2009 WHO clinical practice guideline for dengue [21]. CK, AST, ALT, Hct, platelet count, WBC and serum albumin were taken upon ward admission. As per the recommendations in the guidelines, full blood count was repeated at 8-hourly or 6-hourly intervals. Other blood investigations were repeated at least daily or more frequently as required.

\section{Case definitions for dengue}

Case definitions for the diagnosis of dengue infection and classification of disease severity were based on the 2009 WHO guidelines. According to that system, a suspected dengue case is defined by the presence of fever plus any two of 1) aches and pain, 2) nausea and/or vomiting, 3) rash, 4) leucopenia, or 5) presence of any warning signs. Warning signs are defined as any one of: 1) abdominal pain or tenderness; 2) persistent vomiting (defined as vomiting a minimum of 3 times within $24 \mathrm{~h}$ ); 3) non-physiological or supraphysiological bleeding; 4) lethargy; 5) clinical fluid accumulation that manifest as pleural effusion, ascites 6) a palpable liver $2 \mathrm{~cm}$ below the costal margin; and 7) an elevated hematocrit to $>40 \%$ in female and $>46 \%$ in male, which correspond to an increase of at least $20 \%$ in hematocrit from a genderspecific mean population baseline.

Severe dengue was defined by any of: 1) decompensated shock due to severe plasma leakage (defined as systolic blood pressure of $<90 \mathrm{mmHg}$, a fall of $\geq 40 \mathrm{mmHg}$ from a previous known baseline, or mean arterial blood pressure $<65 \mathrm{mmHg}$ ), 2) compensated shock due to severe plasma leakage (defined as clinical manifestations of reduced perfusion in presence of systolic blood pressure of $\geq 90 \mathrm{mmHg}$ ), 3) respiratory compromise due to severe plasma leakage, 4) severe hepatitis (defined as AST level > $1000 \mathrm{IU} / \mathrm{L}$, or ALT level > $1000 \mathrm{IU} / \mathrm{L}), 5$ ) severe bleeding that required intervention, or 6) severe organ involvement such as acute kidney injury defined by elevated serum creatinine, myocarditis or encephalopathy.

\section{Serology for dengue viral infection}

All sera were tested for the presence of NS1 antigen and high-titre serum IgG. These were processed using the ELISA method (PanBio reagents). Our IgG is a high-titre IgG that is positive at titre $>1: 2560$. IgM was not used in our study for 2 reasons. Firstly, it may remain positive weeks beyond an acute infection hence yielding a false-positive in a non-dengue infection, and secondly, to avoid false positive cases due to detection of cross-reacting antibodies.

\section{Measurement of biomarkers}

As per standard practice at our centre, biomarkers were collected into appropriate Vacutainers ${ }^{\circ}$. CK samples were analyzed using the ultraviolet method, liver transaminases analyzed with International Federation of Clinical Chemistry method (without pyridoxal phosphate activation) and serum albumin were analyzed with bromocresol green (Roche Diagnostics, Malaysia). Platelets were analyzed with hydrodynamic focusing or direct current method, white blood count by flow cytometry and hemoglobin by photometrics (Sysmex, Malaysia).

\section{Composite indices}

We formulated and tested several composite indices based on admission AST and admission ALT values in the search for a marker/model with good performance parameters that could help to better identify severe dengue. Composite indices, like body mass index and shock index [23], have been well-established in clinical use. We utilized simple and common mathematical operators e.g. squaring, division and multiplication in the formation of the indices. Simplicity was the core criterion in formulating these indices to allow for ease of clinical use. Fourteen indices were tested but only one, based on ease of calculation by the bedside, was further scrutinized. Though it is known that liver enzymes would vary along the course of illness, we made no attempt to adjust for this variation as it would only complicate deploying its use to the bedside.

\section{Statistical analysis}

In a previous study [11], when investigators classified dengue into severe and non-severe dengue according to WHO 2009 classification, AUC of ROC curve obtained for AST was 0.62 (95\% CI 0.57 - 0.67). Based on this information, we calculated that a sample size of 19 severe cases was needed for this study, estimating AUC of $72 \%$, confidence level of $95 \%$ and 
power of $80 \%$. No similar information was available for creatine kinase.

All data were analyzed using SPSS software (version 18.0; SPSS Inc., Chicago, IL). Continuous variables were tested for uniformity using the KolmogorovSmirnov test and normality with the Shapiro-Wilk test. Categorical variables were expressed as frequencies and percentages, and comparisons analyzed using the Chi-square test or Fisher's exact test, as appropriate. As our data were mostly non-parametric, we used non-parametric analyses for data interrogation. Continuous variables with non-normal distribution were summarized as median and inter-quartile range (IQR) and comparisons were made using the MannWhitney $\mathrm{U}$ test. Correlation analyses to assess strengths of relationships between biomarkers were made by computing Spearman $r$. Predictive associations between biomarkers were analyzed using regression methods. The method yielding the highest $R^{2}$ was then reported.

Areas under curve of receiver-operating characteristic curves (AUROC) of all models (the composite indices, AST, ALT, CK and Hct) to identify at admission the occurrence of severe dengue, with their 95\% confidence interval, were computed. As an alternative to validation using a second sample in assessing these models to identify severe dengue upon admission, we used the cross-validation technique. Cross-validation will avoid over-fitting of the models thus ensuring accuracy and validity.

The most accurate marker was then assessed further for its performance at four different cut-off values to achieve: 1) screening (cut-off 1), 2) optimal performance/Youden index (cut-off 2), 3) modified Youden to achieve higher specificity but maintain sensitivity $>40 \%$ (cut-off 3), and 4) best diagnostic accuracy (cut-off 4). We assumed that these cut-offs would bear clinical relevance. Sensitivity, specificity, positive predictive value $(\mathrm{PPV})$, negative predictive value (NPV), positive likelihood ratio $(\mathrm{LR}+)$, negative likelihood ratio (LR-) and diagnostic accuracy, with their 95\% confidence intervals were calculated for that best marker. All tests of significance were 2 -sided, and we took $p$-value $<0.05$ to indicate statistical significance.

\section{Results}

\section{Patient characteristics}

A total of 643 patients with suspected dengue who were admitted to the Department of Medicine, Kuala Lumpur Hospital between December 2014 and January 2015 were identified. Of this, patients were excluded for being NS1 antigen and high-titre IgG negative (169 patients) and younger than 18 years old (109 patients). The final number of eligible patients for final analysis was 365 . The admission and in-ward characteristics of the patients are as shown in Tables 1 and 2. Among the patients, 28.9\% were obese $\left(\right.$ BMI $\left.\geq 27.5 \mathrm{~kg} / \mathrm{m}^{2}\right)$. Elevated admission levels of CK, AST, ALT and Hct occurred in 57.1\%, $82.7 \%, 57.5 \%$ and $21.6 \%$ of patients, respectively. Elevated peak levels of CK, AST and ALT occurred in $80.6 \%, 92.2 \%$ and $81.9 \%$ of patients, respectively.

\section{Severe vs non-severe dengue}

In our cohort, $22(6 \%)$ patients had severe dengue, including 1 (4.5\%) patient with decompensated shock, 11 (50\%) with compensated shock, 2 (9.1\%) with respiratory compromise, 8 (36.4\%) with severe hepatitis, 2 (9.1\%) with acute kidney injury and 1 (4.5\%) with encephalitis.

There were some differences in the characteristics of patients with severe and non-severe dengue (see Tables 1 and 2). Patients with severe dengue were significantly more likely to be female, $72.7 \%$ vs $39.1 \%, p=0.003$. These patients were also more likely to have co-morbidities, $36.4 \%$ vs. $13.7 \%$, $p=0.009$, and were more likely to have diabetes mellitus $13.6 \%$ vs. $2.6 \%, p=0.03$. Patients with severe dengue were more likely to have at least 1 co-morbidity ( $p=0.003)$ but there was no significant difference in terms of presence of multiple comorbidities $(p=0.06)$. Patients with severe dengue had significantly higher admission and peak levels of AST and ALT, with peak levels being higher than admission levels. Nadir levels of platelet and serum albumin were also lower in patients with severe dengue.

\section{Diagnostic value of CK, AST and ALT for identifying severe dengue}

We evaluated the diagnostic value of CK, AST, ALT, Hct and the 14 formulated composite indices for identifying severe dengue at admission. Overall, the composite index $\mathrm{AST}^{2} / \mathrm{ALT}$ was the best performing marker in identifying severe dengue on admission. The AUROC for liver enzymes were moderate, with AST being better than ALT, whilst the AUROC for CK and Hct were poor (Table 3). The four cut-off values for $\mathrm{AST}^{2} / \mathrm{ALT}$ were: 98.1 (screening/highest sensitivity cut-off), 402.5 (optimal/Youden index), 653.2 (modified Youden index) and 1193 (highest diagnostic accuracy) (Table 4).

\section{Correlation and predictive associations between markers}

Strength of relationships between markers as determined by Spearman $r$ and their respective predictive associations as represented by $R^{2}$ are shown in Table 5 . AST and ALT showed excellent correlation with each 
Table 1 Baseline characteristics and clinical parameter of 365 patients hospitalized with dengue

\begin{tabular}{|c|c|c|c|c|c|c|c|}
\hline & \multicolumn{2}{|l|}{ All } & \multicolumn{2}{|c|}{ Severe Dengue } & \multicolumn{2}{|c|}{ Non-severe Dengue } & \multirow[t]{2}{*}{$p$-value } \\
\hline & $\bar{n}$ & No. (\% or IQR) & $\bar{n}$ & No. (\% or IQR) & $\bar{n}$ & $\overline{\text { No. }(\% \text { or IQR) }}$ & \\
\hline Gender & 365 & & & & & & $0.003^{a}$ \\
\hline Male & & 215 (58.90\%) & 22 & $6(2.80 \%)$ & 343 & 209 (97.20\%) & \\
\hline Female & & 150 (41.10\%) & 22 & $16(10.70 \%)$ & 343 & 134 (89.30\%) & \\
\hline Age $(y)$, median (IQR) & 364 & $28(23-36)$ & 22 & $31(23-36)$ & 342 & $28(23-36)$ & $0.660^{b}$ \\
\hline BMI, median (IQR) & 353 & $24(21-28)$ & 18 & $25(21-29)$ & 335 & $24(21-28)$ & $0.700^{b}$ \\
\hline Co-morbids present & 365 & $55(15.10 \%)$ & 22 & $8(36.40 \%)$ & 343 & 47 (13.70\%) & $0.009^{a}$ \\
\hline$D M$ & 365 & $12(3.30 \%)$ & 22 & $3(13.60 \%)$ & 343 & $9(2.60 \%)$ & $0.003^{a}$ \\
\hline Hypertension & 365 & $16(4.40 \%)$ & 22 & $3(13.60 \%)$ & 343 & $13(3.80 \%)$ & $0.006^{a}$ \\
\hline Other co-morbids & 365 & 49 (13.40\%) & 22 & $6(27.30 \%)$ & 343 & $43(12.50 \%)$ & $0.100^{a}$ \\
\hline Number of co-morbids & 365 & & 22 & & 343 & & $0.003^{c}$ \\
\hline 0 & & 308 (84.40\%) & 22 & $14(63.60 \%)$ & 343 & 294 (85.70\%) & \\
\hline 1 & & $41(11.20 \%)$ & 22 & $5(22.70 \%)$ & 343 & $36(10.50 \%)$ & \\
\hline 2 & & $12(3.30 \%)$ & 22 & $2(9.10 \%)$ & 343 & $10(2.90 \%)$ & \\
\hline 3 & & $4(1.10 \%)$ & 22 & $1(4.50 \%)$ & 343 & $3(0.90 \%)$ & \\
\hline More than 1 co-morbids present & 365 & $16(4.40 \%)$ & 22 & $3(13.60 \%)$ & 343 & $13(3.80 \%)$ & $0.006^{a}$ \\
\hline
\end{tabular}

${ }^{a}$ Fisher's exact test or Chi-square test, as appropriate

${ }^{b}$ Mann-Whitney $U$ test

${ }^{c}$ Chi-square for trend

$I Q R$ interquartile range, $B M I$ body mass index, $D M$ diabetes mellitus

other, at admission and at peak values. In addition, admission AST showed a good predictive association with admission ALT $\left(R^{2} 0.79\right)$ but the association weakened at peak values $\left(R^{2} 0.66\right)$ despite better correlation. The correlation between liver enzymes and plasma leakage markers, hematocrit and serum albumin were weak to negligible, $r<0.10$ and $<-0.40$. Liver enzymes also showed very poor predictive associations with nadir platelet and nadir serum albumin, $R^{2} 0.003-0.09$.

CK showed a weak to negligible correlation with liver enzymes at both admission and peak values $(r 0.11$ 0.37) (Table 5). The correlations between CK and plasma leakage markers, hematocrit and serum albumin were negligible $(r<0.30$ and $<-0.30)$. Similarly, the correlations between CK and disease course marker, platelet count, were also negligible $(r<-0.30)$. CK showed very poor predictive associations with liver enzymes, leakage markers and disease course marker $\left(R^{2} 0.001-0.13\right)$.

Interestingly, we also found that there was a negligible correlation ( $r \quad 0.19$ (95\% CI: 0.08 - 0.29 ), $p=0.001)$ and a non-existent predictive association $\left(\begin{array}{ll}R^{2} & 0.03\end{array}\right)$ between admission Hct and nadir serum albumin (Table 5).

\section{Discussions}

Our analysis showed that admission CK was not helpful in identifying severe dengue and does not correlate with any of the biomarkers which represent hepatitis (ALT), plasma leakage (hematocrit and serum albumin) and disease course (platelet count). Musculoskeletal involvement in dengue takes the form of myalgia and arthralgia. It was demonstrated in a cohort of 1716 patients in Latin America that this occurred in $94.3 \%$ of their patients [24]. We emphasize again that our study focused on biomarkers at admission - when it would be most relevant time to predict development of severe dengue. Also, as mentioned, our case definitions used the current WHO 2009 guidelines. In Villar-Centeno et al. (2008), the values of CK in the dengue haemorrhagic fever (DHF, WHO 1997) group were mean (95\% CI): 549.6 (267.5-831.6) U/L taken 2-4 days into the illness. Our study showed admission $\mathrm{CK}$ in the severe dengue group are media$n(I Q R): 212$ (116 - 300) U/L taken 3-5 days into the illness. The peak CK values in our study were 404 (171 - 766) U/L. The difference between non-severe and severe groups were not significant $(p=0.43)$. It seems therefore that although musculoskeletal involvement occurred in almost all dengue patients, its significance beyond symptomatology is unclear. Further studies are needed to demonstrate the clinical relevance of monitoring CK.

However, our study revealed that, at admission, the identification of severe dengue was best using the composite index, $\mathrm{AST}^{2} / \mathrm{ALT}$, based on AST and ALT readings taken at admission. The AUROC was 0.83, which makes it to date one of the two markers with 


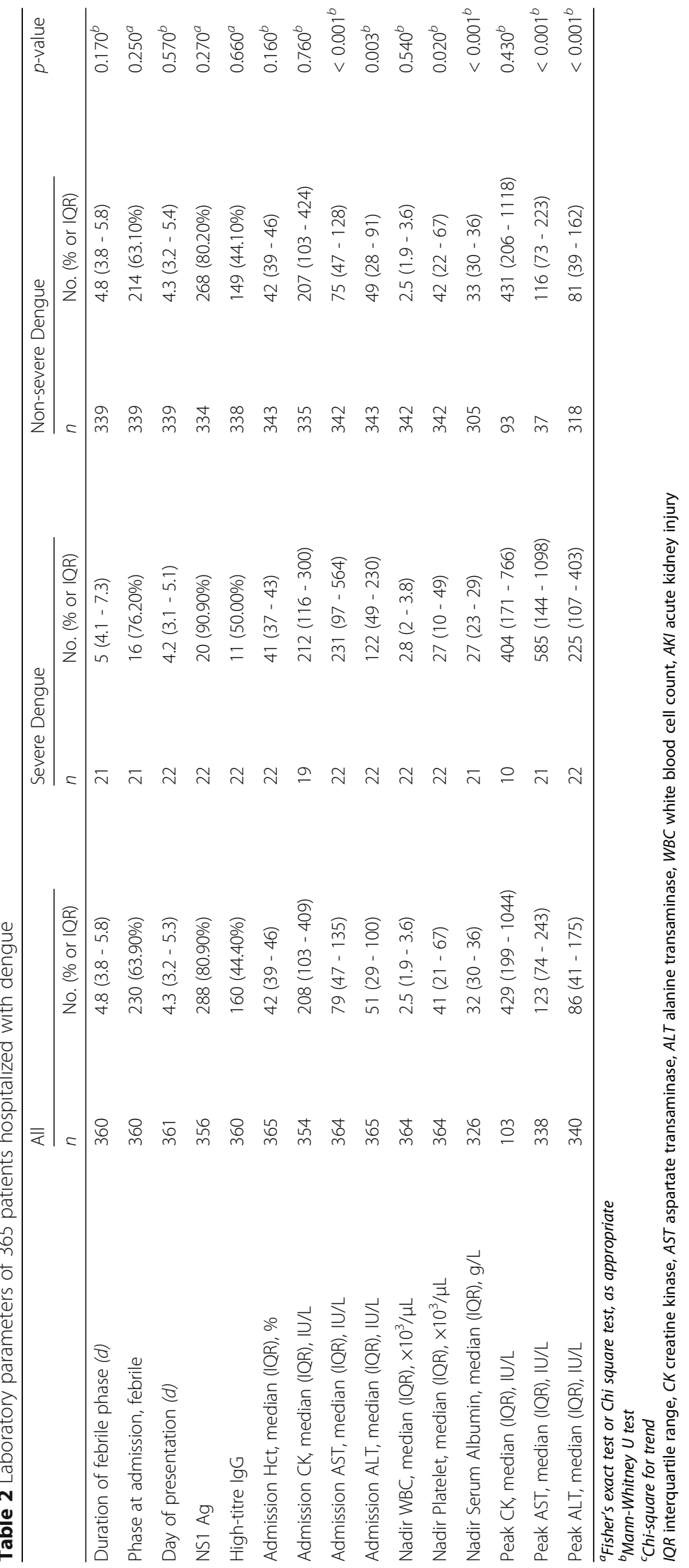


Table 3 Area under curve (AUC) of CK, AST, ALT, Hct, AST $2 / A L T$ and 13 other composite indices

\begin{tabular}{|c|c|}
\hline Markers & AUC (95\% Cl) \\
\hline CK & $0.52(0.40-0.64)$ \\
\hline AST & $0.78(0.68-0.89)$ \\
\hline ALT & $0.69(0.56-0.81)$ \\
\hline Hct & $0.59(0.47-0.71)$ \\
\hline $\mathrm{AST}^{2} / \mathrm{ALT}$ & $0.83(0.73-0.92)$ \\
\hline AST/ALT & $0.77(0.67-0.87)$ \\
\hline $\mathrm{AST} \times \mathrm{ALT}$ & $0.74(0.62-0.86)$ \\
\hline $\mathrm{AST}^{2} \times \mathrm{ALT}$ & $0.76(0.64-0.87)$ \\
\hline AST $\times \sqrt{ }$ ALT & $0.76(0.64-0.87)$ \\
\hline$\sqrt{ } A S T \times A L T$ & $0.72(0.60-0.84)$ \\
\hline AST/VALT & $0.83(0.73-0.92)$ \\
\hline$\sqrt{ }$ AST/ALT & $0.42(0.30-0.55)$ \\
\hline $\mathrm{AST}^{2} / \sqrt{ } \mathrm{ALT}$ & $0.81(0.71-0.90)$ \\
\hline $\mathrm{AST}^{2} / \mathrm{ALT}^{3}$ & $0.52(0.41-0.63)$ \\
\hline $\mathrm{AST}^{3} / \mathrm{ALT}$ & $0.81(0.72-0.91)$ \\
\hline $\mathrm{AST}^{3} / \sqrt{ } \mathrm{ALT}$ & $0.80(0.69-0.90)$ \\
\hline $\mathrm{AST}^{3} / \mathrm{ALT}^{2}$ & $0.83(0.73-0.93)$ \\
\hline $\mathrm{AST}^{4} / \mathrm{ALT}^{3}$ & $0.82(0.73-0.92)$ \\
\hline
\end{tabular}

AUC area under curve; $A S T$ aspartate transaminase; $A L T$ alanine transaminase; $\mathrm{Hct}$ hematocrit; $C K$ creatine kinase; $C l$ confidence interval

highest diagnostic performance. Our composite index requires, however, the performance of liver function tests and AST. At our hospital liver function tests and AST are available on an urgent basis with a short turnaround time. The simplicity of the formula allows for ease of clinical use. We propose 2 cut-offs, 402 and 653, that will help to delineate severe dengue. Such suspicion may arise in those who have, for example, isolated tachycardia in the presence of high grade fever, isolated single manifestation of peripheral sign of shock, a remarkable isolated derangement of hematocrit or isolated mild metabolic acidosis. Choice of cut-off will also depend on clinical resources available, with the higher value more suitable if resources are limited.

In addition, our correlational analysis suggests that AST originates from the liver rather than muscles. AST has been showed to rise earlier and to a higher level than ALT in almost all dengue patients [12]. This makes AST a better marker to monitor the liver and identify severe hepatitis earlier. Its levels also decline earlier than ALT [8], making it well-suited in monitoring the course of hepatitis. In comparison to other biomarkers and composite indices studied, the diagnostic performance of AST was second only to $\mathrm{AST}^{2} / \mathrm{ALT}$, with AUROC of 0.78. Thus, it is better than the traditional ALT in monitoring hepatitis in dengue. Our results of the diagnostic performances of the liver enzymes are better than a previous study [11] because we included severe hepatitis as part of the outcome of severe dengue. We believe it is important to be able to identify the development of severe hepatitis at an earlier stage albeit without the patient manifesting other features of severe dengue.

Our correlational analysis found that the pathophysiological processes that occur in dengue, represented by the different biomarkers studied, do not correlate with each other, confirming our anecdotal observation. This suggests that these processes occur independently of one another in dengue. Adding to the puzzle is our findings that markers of plasma leakage, Hct and serum albumin, do not correlate with each other. The sampling time points of these biomarkers in our study were different. Nonetheless, two markers representing the same disease process ought to have a better degree of correlation. Furthermore, the sampling time points chosen in our analysis were appropriate for each hematocrit and serum albumin. Admission Hct would not be influenced by the later clinical administration of fluid and may be assumed to be the highest before intervention was instituted. Similarly, we used nadir serum albumin which may reflect peak leakage and is unaffected by fluid administration. This paradox needs further evaluation.

The main limitation of our study was that it was retrospective. However, data accuracy was reasonable as management of patients followed standard local management guidelines for dengue with clear specifications of timing of blood investigations. Our second limitation was that we did not perform serotyping. The dominant serotypes during the time of this study were Den-1 at approximately $55 \%$ of national surveillance cases and Den-2 at approximately $35 \%$. Den-3 and Den-4 each contributed to less than $10 \%$ of cases. Since mid-2013 Den-1 and Den-2 have been the predominant serotypes in Malaysia [25]. Third, although we suggested that AST was of liver origin based on the strong correlation with ALT and negligible correlation with $\mathrm{CK}$, we did not test for other sources of AST such as brain, red blood cells and kidney. However, none of our patient had hemolysis, only 2 patients had increments in serum creatinine and only one had encephalitis. Fourth, we did not perform multivariate analysis on our composite indices. A multivariate analysis would require a larger number of patients with severe dengue, which in turn would be determined by the number of covariates included. Finally, we did not adjust for the usage of paracetamol and traditional medication which is prevalent in our population, which could 


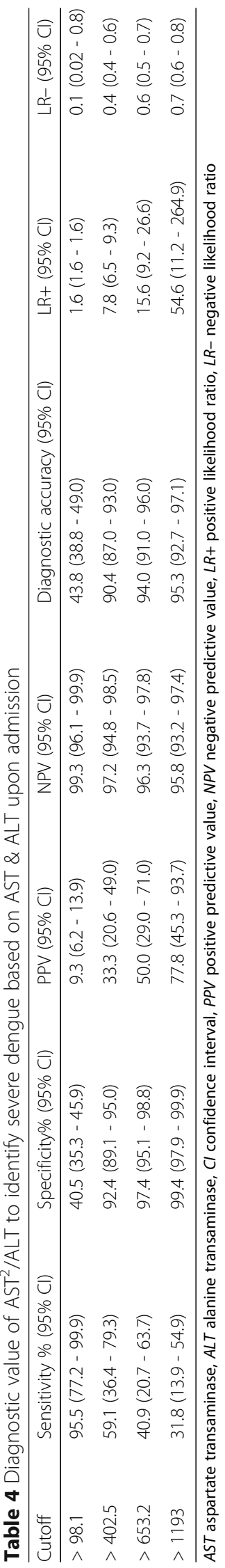


Table 5 Correlation coefficients and coefficients of determination for CK, AST, ALT, markers of plasma leakage and platelet count

\begin{tabular}{|c|c|c|c|c|}
\hline & $n$ & Spearman $r(95 \% \mathrm{Cl})$ & $p$ & $R^{2}$ \\
\hline Admission CK - Admission AST & 364 & $0.37(0.27-0.46)$ & $<0.001$ & $0.13^{\mathrm{a}}$ \\
\hline Admission CK - Peak AST & 206 & $0.28(0.15-0.41)$ & $<0.001$ & $0.07^{\mathrm{a}}$ \\
\hline Peak CK - Admission AST & 104 & $0.29(0.09-0.46)$ & 0.003 & $0.06^{\mathrm{a}}$ \\
\hline Peak CK - Peak AST & 92 & $0.22(0.03-0.39)$ & 0.020 & $0.06^{\mathrm{a}}$ \\
\hline Admission CK - Admission ALT & 364 & $0.26(0.16-0.35)$ & $<0.001$ & $0.02^{\mathrm{b}}$ \\
\hline Admission CK - Peak ALT & 216 & $0.11(-0.02-0.24)$ & 0.110 & $0.01^{b}$ \\
\hline Peak CK - Admission ALT & 217 & $0.25(0.06-0.42)$ & 0.010 & $0.05^{c}$ \\
\hline Peak CK - Peak ALT & 217 & $0.12(-0.08-0.32)$ & 0.240 & $0.02^{c}$ \\
\hline Admission CK - Admission Hct & 364 & $0.24(0.14-0.33)$ & $<0.001$ & $0.01^{c}$ \\
\hline Admission CK - Nadir Platelet & 363 & $-0.22(-0.32--0.12)$ & $<0.001$ & $0.01^{c}$ \\
\hline Admission CK - Nadir Serum Albumin & 326 & $-0.04(-0.15-0.07)$ & 0.500 & $0.001^{\mathrm{C}}$ \\
\hline Peak CK - Admission Hct & 104 & $0.03(-0.17-0.22)$ & 0.800 & $0.004^{c}$ \\
\hline Peak CK - Nadir Platelet & 104 & $-0.10(-0.29-0.09)$ & 0.300 & $0.01^{c}$ \\
\hline Peak CK - Nadir Serum Albumin & 104 & $0.06(-0.13-0.25)$ & 0.540 & $0.01^{c}$ \\
\hline Admission AST - Admission ALT & 365 & $0.86(0.82-0.88)$ & $<0.001$ & $0.79^{\mathrm{b}}$ \\
\hline Admission AST - Peak ALT & 217 & $0.68(0.60-0.75)$ & $<0.001$ & $0.54^{\mathrm{b}}$ \\
\hline Peak AST - Admission ALT & 206 & $0.66(0.57-0.73)$ & $<0.001$ & $0.45^{\mathrm{a}}$ \\
\hline Peak AST - Peak ALT & 189 & $0.92(0.89-0.94)$ & $<0.001$ & $0.66^{b}$ \\
\hline Admission Hct - Nadir Platelet & 365 & $-0.14(-0.24--0.03)$ & 0.009 & $0.06^{c}$ \\
\hline Admission Hct - Nadir Serum Albumin & 327 & $0.19(0.08-0.29)$ & 0.001 & $0.03^{c}$ \\
\hline Nadir Serum Albumin - Nadir Platelet & 327 & $0.52(0.43-0.60)$ & $<0.001$ & $0.14^{\mathrm{b}}$ \\
\hline Admission AST - Admission Hct & 365 & $0.05(-0.06-0.15)$ & 0.380 & $0.006^{\mathrm{C}}$ \\
\hline Admission AST - Nadir Platelet & 365 & $-0.34(-0.43--0.25)$ & $<0.001$ & $0.04^{b}$ \\
\hline Admission AST - Nadir Serum Albumin & 327 & $-0.32(-0.41--0.21)$ & $<0.001$ & $0.09^{c}$ \\
\hline Peak AST - Admission Hct & 206 & $-0.02(-0.15-0.12)$ & 0.800 & $0.01^{c}$ \\
\hline Peak AST - Nadir Platelet & 206 & $-0.38(-0.49--0.26)$ & $<0.001$ & $0.08^{c}$ \\
\hline Peak AST - Nadir Serum Albumin & 200 & $-0.29(-0.41--0.16)$ & $<0.001$ & $0.09^{c}$ \\
\hline Admission ALT - Admission Hct & 365 & $0.09(-0.06-0.15)$ & 0.080 & $0.003^{\mathrm{C}}$ \\
\hline Admission ALT - Nadir Platelet & 365 & $-0.19(-0.43--0.25)$ & $<0.001$ & $0.03^{c}$ \\
\hline Admission ALT - Nadir Serum Albumin & 327 & $-0.16(-0.41--0.21)$ & 0.003 & $0.04^{c}$ \\
\hline Peak ALT - Admission Hct & 217 & $-0.02(-0.06-0.15)$ & 0.810 & $0.01^{c}$ \\
\hline Peak ALT - Nadir Platelet & 217 & $-0.17(-0.43--0.25)$ & 0.020 & $0.01^{c}$ \\
\hline Peak ALT - Nadir Serum Albumin & 209 & $-0.13(-0.41--0.21)$ & 0.050 & $0.03^{c}$ \\
\hline
\end{tabular}

${ }^{\text {a Power regression }}$

binear regression

'Polynomial regression;

CK creatine kinase, AST aspartate transaminase, ALT alanine transaminase, Hct hematocrit

have been one of the causes of elevated transaminases. We have focused and limited our discussion to biomarkers examined in our study as per objective. There are of course many other known risk factors that are known to be associated with development of severe dengue, beyond the scope of our study. A larger study that examines extensively all potential risk factors together would be suited to review these.

\section{Conclusion}

We conclude that $\mathrm{AST}^{2} / \mathrm{ALT}$ may be used as a marker to identify severe dengue based on admission AST and ALT, with two choices of cut-off values, 402 and 653 .

\section{Abbreviations}

ALT: Alanine aminotransferase; AST: Aspartate aminotransferase; AUROC: Area under the receiver operating characteristic; $\mathrm{Cl}$ : Confidence interval;

CK: Creatine kinase; ELISA: Enzyme-linked immunosorbent assays;

Hct: Hematocrit; IgG: Immunoglobulin G; IgM: Immunoglobulin M; IQR: Inter- 
quartile range; LR-: Negative likelihood ratio; $L R+$ : Positive likelihood ratio; NPV: Negative predictive value; NS1: Non-structural protein-1; PPV: Positive predictive value; ROC: Receiver operating characteristic; STARD: Standards for reporting of diagnostic accuracy; WBC: White blood cell count; WHO: World Health Organization.

\section{Acknowledgements}

The authors would like to thank all the staff and nurses in the Department of Medicine and the Records Office, Kuala Lumpur Hospital. We would also like to thank Dr. Yen Yew Ngau (Head, Department of Medicine, Kuala Lumpur Hospital), Dr. Noel Thomas Ross (Deputy Head, Department of Medicine, Kuala Lumpur Hospital) and Dr. Fatt Soon Lee for their appraisals and support. We would like to thank Ms. Khairul Bariyyah Mohd Hatta for her assistance in technical editing of the manuscript.

\section{Funding}

This research did not receive any specific grants from funding agencies in the public, commercial, or not-for-profit sectors.

\section{Availability of data and materials}

The data that support the findings of this study are available from the Kuala Lumpur Hospital but restrictions apply to the availability of these data, which were used under license for the current study, and so are not publicly available. Data are however available from the authors upon reasonable request and with permission of the Kuala Lumpur Hospital.

\section{Authors' contributions}

SSMS contributed to the conception, study design, data analysis, data interpretation and drafting the article; HWH contributed to data collection, data interpretation, drafting the article and management of dengue patients; BMA contributed to the data analysis, data interpretation and drafting the article; DHJ contributed to data interpretation, drafting the article and management of dengue patients; NKL contributed to data collection and management of dengue patients; MAAS contributed to the study design, study registration and ethical approval. All authors read and approved the final manuscript.

\section{Ethics approval and consent to participate}

The study was approved by the Malaysian Research Ethics Committee, Ministry of Health of Malaysia (Research ID NMRR-14-1543-23635). Our report is based on the Standards for Reporting of Diagnostic Accuracy (STARD) 2015 guideline. This was a retrospective cohort study of adult patients with confirmed dengue infection who were admitted to Kuala Lumpur Hospital and hence informed consent was not necessary for this study.

\section{Consent for publication}

Not applicable.

\section{Competing interests}

The authors declare that they have no competing interests.

\section{Publisher's Note}

Springer Nature remains neutral with regard to jurisdictional claims in published maps and institutional affiliations.

\section{Author details \\ 'Department of Medicine, Hospital Kuala Lumpur, Jalan Pahang, 50586 Kuala Lumpur, Malaysia. ${ }^{2}$ Biostatistic Unit, National Clinical Research Centre, 1st Floor, MMA Building, 124 Jalan Pahang, 53000 Kuala Lumpur, Malaysia. ${ }^{3}$ Clinical Research Centre, Hospital Kuala Lumpur, Jalan Pahang, 50586 Kuala Lumpur, Malaysia.}

Received: 15 September 2016 Accepted: 14 July 2017

Published online: 21 July 2017

\section{References}

1. Hales S, de Wet N, Maindonald J, Woodward A. Potential effect of population and climate changes on global distribution of dengue fever: an empirical model. Lancet. 2002;360:830-4. doi:10.1016/S0140-6736(02)09964-6.
2. Kenyataan Akhbar Ketua Pengarah Kesihatan Untuk Denggi Minggu 47/ 2014. http://www.moh.gov.my/index.php/database_stores/store_view page/17/596. Accessed 2 Dec 2014

3. Misra UK, Kalita J, Maurya PK, Kumar P, Shankar SK, Mahadevan A. Dengue-associated transient muscle dysfunction: clinical, electromyography and histopathological changes. Infection. 2012:40(2): 125-30. doi:10.1007/s15010-011-0203-8.

4. Villar-Centeno LA, Díaz-Quijano FA, Martínez-Vega RA. Biochemical alterations as markers of dengue hemorrhagic fever. Am J Trop Med Hyg. 2008;78(3):370-4

5. Souza LJD, Alves JG, Nogueira RMR, Gicovate Neto C, Bastos DA, Siqueira EWDS, et al. Aminotransferase changes and acute hepatitis in patients with dengue fever: analysis of 1,585 cases. Braz J Infect Dis. 2004;8(2):156-63. doi: 10.1590/S1413-86702004000200006.

6. Nguyen TL, Nguyen TH, Tieu NT. The impact of dengue haemorrhagic fever on liver function. Res Virol. 1997:148(4):273-7.

7. Guilarde AO, Turchi MD, Siqueira JB Jr, Feres VC, Rocha B, Levi JE, et al. Dengue and dengue hemorrhagic fever among adults: clinical outcomes related to viremia, serotypes, and antibody response. J Infect Dis. 2008:197(6):817-24. doi:10.1086/528805.

8. Hien TT, Vinh NN, Hien PTD, Chinh NT, Simmons C, Wills B. Liver involvement associated with dengue infection in adults in Vietnam. Am J Trop Med Hyg. 2010;83(4):774-80. doi:10.4269/ajtmh.2010.10-0090.

9. Kalayanarooj S, Vaughn DW, Nimmannitya S, Green S, Suntayakorn S, Kunentrasai N, et al. Early clinical and laboratory indicators of acute dengue illness. J Infect Dis. 1997;176(2):313-21. doi:10.1086/514047.

10. Raza FA, Rehman S, Khalid R, Ahmad J, Ashraf S, lqbal M, et al. Demographic and Clinico-epidemiological features of dengue fever in Faisalabad, Pakistan. PLoS ONE. 2014;9(3):e89868. doi:10.1371/journal. pone.0089868.

11. Lee LK, Gan VC, Lee VJ, Tan AS, Leo YS, Lye DC. Clinical relevance and discriminatory value of elevated liver Aminotransferase levels for dengue severity. PLoS Negl Trop Dis. 2012:6(6):e1676. doi:10.1371/ journal.pntd.0001676.

12. Krishnamurti C, Kalayanarooj S, Cutting MA, Peat RA, Rothwell SW, Reid TJ, et al. Mechanisms of hemorrhage in dengue without circulatory collapse. Am J Trop Med Hyg. 2001;65(6):840-7.

13. Kuo CH, Tai DI, Chang-Chien CS, Lan CK, Chiou SS, Liaw YF. Liver biochemical tests and dengue fever. Am J Trop Med Hyg. 1992;47(3):265-70.

14. Thanachartwet V, Oer-Areemitr N, Chamnanchanunt S, Sahassananda D, Jittmittraphap A, Suwannakudt P, et al. Identification of clinical factors associated with severe dengue among Thai adults: a prospective study. BMC Infect Dis. 2015;15(1):1. doi:10.1186/s12879-015-1150-2.

15. Thein T-L, Leo Y-S, Fisher DA, Low JG, Oh HML, Gan VC, et al. Risk factors for fatality among confirmed adult dengue inpatients in Singapore: a matched case-control study. PLoS One. 2013;8(11):e81060. doi:10.1371/journal.pone.0081060

16. Jayaratne SD, Atukorale V, Gomes L, Chang T, Wijesinghe T, Fernando S, et al. Evaluation of the WHO revised criteria for classification of clinical disease severity in acute adult dengue infection. BMC Res Notes. 2012;5:645. doi:10. 1186/1756-0500-5-645.

17. Huy NT, Van Giang T, Thuy DH, Kikuchi M, Hien TT, Zamora J, et al. Factors associated with dengue shock syndrome: a systematic review and metaanalysis. PLoS Negl Trop Dis. 2013;7(9):e2412. doi:10.1371/journal.pntd.0002412.

18. World Health Organization. Dengue haemorrhagic fever: diagnosis, treatment and control. WHO: Geneva; 1997.

19. Tricou V, Minh NN, Farrar J, Tran HT, Simmons CP. Kinetics of viremia and NS antigenemia are shaped by immune status and virus serotype in adults with dengue. PLoS Negl Trop Dis. 2011;5(9):e1309. doi:10.1371/journal.pntd.0001309.

20. Bossuyt PM, Reitsma JB, Bruns DE, Gatsonis CA, Glasziou PP, Irwig L, et al. STARD 2015: an updated list of essential items for reporting diagnostic accuracy studies. Radiology. 2015;277(3):826-32. doi:10.1148/radiol.2015151516.

21. World Health Organization, Special Programme for Research, Training in Tropical Diseases, World Health Organization. Department of Control of neglected tropical diseases, World Health Organization. Epidemic, \& pandemic alert. Dengue: quidelines for diagnosis, treatment, prevention and control: World Health Organization; 2009

22. Health Technology Assessment Section, Medical Development Division, Ministry of Health Malaysia. Clinical practice guidelines on Management of Dengue Infection in adults revised 2nd edition: Ministry of Health Malaysia; 2010. 
23. Yussof SJ, Zakaria MI, Mohamed FL, Bujang MA, Lakshmanan S, Asaari AH. Value of shock index in prognosticating the short term outcome of death for patients presenting with severe sepsis and septic shock in the emergency department. Med J Malaysia. 2012;67(4):406-11.

24. Halsey ES, Marks MA, Gotuzzo E, Fiestas V, Suarez L, Vargas J, et al. Correlation of serotype-specific dengue virus infection with clinical manifestations. PLoS Negl Trop Dis. 2012;6(5):e1638. doi:10.1371/journal. pntd.0001638.

25. Health Technology Assessment Section, Medical Development Division, Ministry of Health Malaysia. Clinical practice guidelines on Management of Dengue Infection in adults 3rd edition: Ministry of Health Malaysia; 2015.

Submit your next manuscript to BioMed Central and we will help you at every step:

- We accept pre-submission inquiries

- Our selector tool helps you to find the most relevant journal

- We provide round the clock customer support

- Convenient online submission

- Thorough peer review

- Inclusion in PubMed and all major indexing services

- Maximum visibility for your research

Submit your manuscript at www.biomedcentral.com/submit
C Biomed Central 